\title{
APPLICATIONS OF SELECTION THEOREMS TO RADIAL CLUSTER SET INTERPOLATION FOR FUNCTIONS ON THE UNIT BALL
}

\author{
Robert D. Berman and Togo Nishiura
}

\begin{abstract}
For $B_{n}=\{\|x\|<1\}$ and $S^{n-1}=\{\|x\|=1\}$ in $R^{n}$ and a compact metric space $Y$, let $f^{\oplus}$ be the radial cluster set function corresponding to a continuous function $f: B_{n} \rightarrow Y$. The function $f^{\oplus}$ maps $S^{n-1}$ into the metric space $\mathcal{C}(Y)$ of continua in $Y$, where the metric is the Hausdorff metric. Some topological properties of $f^{\oplus}$ were investigated by F. Bagemihl with W. Seidel in [2] and [3], and with J.E. McMillan in [1] when $Y=S^{2}$ and $n=2$. Certain problems left unresolved are easily reduced to "interpolation problems" on the boundary. These interpolation problems are investigated here with the aid of the Borel measurable selection theorems of C.J. Himmelberg, F.S. Van Vleck and K. Prikry [12]. The following is among the theorems proved. If $E$ is an $F_{\sigma}$ subset of $S^{n-1}$ and $\phi: E \rightarrow \mathcal{C}\left(S^{k}\right)(k \geq 2)$ is a Baire class 1 function such that $\infty \notin \phi(\zeta)$ for all $\zeta$ in $E$, then there exists a continuous function $f: B_{n} \rightarrow S^{k} \backslash\{\infty\}$ such that $f^{\oplus}(\zeta)=\phi(\zeta)$ for each $\zeta$ in $E$. ( $\infty$ is a distinguished point of $S^{k}$.) A second theorem shows that the assumption " $\infty \notin \phi(\zeta)$ " can be dropped in exchange for a dimension assumption on $E$. This second theorem will be employed in a follow-up paper to prove a longstanding conjecture of Bagemihl and Seidel [2, p. 99] and to extend a recent result of L.W. Brinn [6].
\end{abstract}

\section{Introduction}

For $n \geq 1$, let $B_{n}$ and $S^{n-1}$ be the open unit ball $\{\|x\|<1\}$ and its bounding sphere $\{\|x\|=1\}$ in $n$-dimensional Euclidean space $R^{n}$; and, let $Y$ be a compact metric space. For a continuous function $f: B^{n} \rightarrow Y$ and a point $\zeta$ in $S^{n-1}$, the radial cluster set of $f$ at $\zeta$, denoted $f^{\oplus}(\zeta)$, is the nonempty continuum in $Y$ consisting of the limit points of all the convergent sequences $\left\{f\left(r_{m} \zeta\right)\right\}$, where $\left\{r_{m}\right\}$ is a real-valued sequence increasing to 1 . This function $f^{\oplus}$ defined on $S^{n-1}$ with values in $\mathcal{C}(Y)$ (the collection of nonempty subcontinua of $Y$ ) is called the radial cluster set function for $f$. The collection $\mathcal{C}(Y)$ is a closed subset of the compact metric space $2^{Y}$ (the collection of nonempty closed subsets of $Y$ ) endowed with the Hausdorff metric which is naturally induced on $2^{Y}$ by the metric of $Y$. (A short discussion of the "hyperspace" $2^{Y}$ is given in Section 3). Some topological properties of $f^{\oplus}$ were investigated by F. Bagemihl and W. Seidel in 1954-1955 ([2] and [3]) and by F. Bagemihl and J.E. McMillan in 1966 [1] for the case of $Y=S^{2}$ (the Riemann sphere $\widehat{C}=C \cup\{\infty\}$ ) of complex variables. These 
earlier investigations had left unresolved certain problems which are easily reduced to interpolation problems. By an interpolation problem, we mean the problem of determining, for a subset $E$ of $S^{n-1}$ and a class $\Phi$ of functions $\phi: E \rightarrow \mathcal{C}(Y)$ (such as a Baire class), whether each function $\phi$ in $\Phi$ is associated with some continuous function $f$ on $B_{n}$ into $Y$ such that $f^{\oplus}$ interpolates $\phi$ in the sense that $f^{\oplus}(\zeta)=\phi(\zeta)$ for each $\zeta$ in $E$. With the appearance of the Borel measurable selection theorems of C.J. Himmelberg, F.S. Van Vleck and K. Prikry [12] in 1985, we are able now to prove the relevant interpolation theorems needed to resolve these problems. The selection theorem that is used in our paper is given below as Theorem 3.5.

Before stating our main theorems, let us set down some notation. Generalizing the complex variable case, we shall let $Y$ be $S^{k}$ with $\infty$ being the point $(0, \ldots, 0,1)$ of $S^{k}(k \geq 1)$. We shall assume that $R^{k}$ is embedded into $R^{k+1}$ in the obvious manner and denote the stereographic projection by $\Pi: S^{k} \backslash\{\infty\} \rightarrow R^{k}$.

The first interpolation theorem concerns the class $\Phi$ of Baire class 1 functions $\phi: E \rightarrow \mathcal{C}\left(S^{k}\right)$ where $E$ is an $F_{\sigma}$-subset of $S^{n-1}$ and $\phi(\zeta)$ does not contain $\infty$ for each $\zeta$ in $E$. Recall that $\phi$ is of Baire class 1 provided it is a pointwise limit (in the Hausdorff metric) of a sequence of continuous functions.

Theorem 1.1. Let $n \geq 1$ and $k \geq 2$. Suppose that $E$ is an $F_{\sigma}$ subset of $S^{n-1}$ and that $\phi: E \rightarrow \mathcal{C}\left(S^{k}\right)$ is a function with $\infty \notin \phi(\zeta)$ for each $\zeta$ in $E$. If $\phi$ is of Baire class 1, then there exists a continuous function $f: B_{n} \rightarrow S^{k} \backslash\{\infty\}$ such that $f^{\oplus}(\zeta)=\phi(\zeta)$ for each $\zeta$ in $E$.

Our next result concerns radial-limit function $f^{*}$ of a continuous function $f: B_{n+1} \rightarrow S^{k} \backslash\{\infty\}$. By definition, $f^{*}$ is the function whose domain consists of the set of points $\zeta$ in $S^{n}$ for which $f^{\oplus}(\zeta)$ is a singleton and whose value $f^{*}(\zeta)$ is the unique point in that singleton set.

Theorem 1.2. If $k \geq 1$ and $h: S^{k} \rightarrow S^{k}$ is a continuous function, then there exists a continuous function $f: B_{k+1} \rightarrow S^{k} \backslash\{\infty\}$ such that $f^{*}=h$.

Note that this shows that the homotopy classes of continuous mappings of $S^{k}$ into itself are not preserved under pointwise convergence.

Our final interpolation theorem relaxes the condition $\infty \notin \phi(\zeta)$ for $\zeta \in E$ that appears in Theorem 1.1. Here, we will need a stronger condition on the $F_{\sigma}$-set $E$, namely $\operatorname{dim} E \leq k-2$. (See Section 4 for a discussion on dimension.)

Theorem 1.3. Let $n \geq 1$ and $k \geq 2$. Suppose that $E$ is an $F_{\sigma}$ subset of $S^{n-1}$ and that $\phi: E \rightarrow \mathcal{C}\left(S^{\bar{k}}\right)$. If $\operatorname{dim} E \leq k-2$ and $\phi$ is of Baire class 1, then there exists a continuous function $f: B_{n} \rightarrow S^{k} \backslash\{\infty\}$ such that $f^{\oplus}(\zeta)=\phi(\zeta)$ for each $\zeta$ in $E$.

The proofs of the above interpolation theorems are made possible by the following characterization of the class of functions $\phi$ defined on subsets $E$ of $S^{n-1}$ 
which are restrictions of some radial cluster set function $f^{\oplus}$. The statements of the theorems will use the following two well-known notions. Suppose that $E$ is a subset of the sphere $S^{n-1}$. For a sequence $\phi_{m}: E \rightarrow 2^{Y}(m=1,2, \ldots)$ of functions defined on the set $E$, we refer to the function $\phi$ as the topological limit superior provided

$$
\phi(\zeta)=\cap_{j} \overline{\cup_{m \geq j} \phi_{m}(\zeta)}, \quad \zeta \in E .
$$

A function $h: E \rightarrow Y$ defined on the boundary set $E$ is called a selection for a function $\phi: E \rightarrow 2^{Y}$ provided $h(\zeta) \in \phi(\zeta)$ when $\zeta \in E$. In case the functions are defined on a set larger than $E$, we use the same terminology with the appended phrase "on $E$ ".

Theorem 1.4. Let $n \geq 1$ and $k \geq 2$. Then, the following two statements hold.

(A) For each continuous function $f: B_{n} \rightarrow S^{k} \backslash\{\infty\}$, there is a sequence of pairs $h_{m}: S^{n-1} \rightarrow S^{k} \backslash\{\infty\}$ and $\phi_{m}: S^{n-1} \rightarrow \mathcal{C}\left(S^{k}\right)(m=1,2, \ldots)$ of continuous functions such that the function $h_{m}$ is a selection for $\phi_{m}$, the function $f^{\oplus}$ is the topological limit superior of the sequence $\left\{\phi_{m}\right\}$, and

$$
\lim _{m \rightarrow \infty} \max \left\{\left\|\Pi \circ h_{m}(\zeta)-\Pi \circ h_{m+1}(\zeta)\right\|: \zeta \in S^{n-1}\right\}=0 .
$$

(B) For each sequence of pairs $h_{m}: S^{n-1} \rightarrow S^{k} \backslash\{\infty\}$ and $\phi_{m}: S^{n-1} \rightarrow \mathcal{C}\left(S^{k}\right)$ $(m=1,2, \ldots)$ of continuous functions such that $h_{m}$ is a selection for $\phi_{m}$ there is a continuous function $f$ on $B_{n}$ into $S^{k} \backslash\{\infty\}$ such that $f^{\oplus}(\zeta)$ contains $\phi(\zeta)$, where $\phi$ is the topological limit superior of the sequence $\left\{\phi_{m}\right\}$, and the condition

$$
\lim _{m \rightarrow \infty}\left\|\Pi \circ h_{m}(\zeta)-\Pi \circ h_{m+1}(\zeta)\right\|=0
$$

implies that $f^{\oplus}(\zeta)=\phi(\zeta)$.

The proof of statement (B) of the above theorem is carried out by piecing together specially constructed homotopies.

In the above mentioned paper of Bagemihl and McMillan [1], a natural notion of "uniform convergence of $f$ to $f^{\oplus}$ " is defined (see Section 3 for a precise definition). The next theorem characterizes those set-valued functions $\phi$ defined on the boundary $S^{n-1}$ for which some continuous function $f: B_{n} \rightarrow S^{k} \backslash\{\infty\}$ converges uniformly to $\phi$ in the sense of Bagemihl and McMillan.

Theorem 1.5. Let $n \geq 1, k \geq 2$ and let $\phi: E \rightarrow 2^{S^{k}}$ be a function defined on a subset $E$ of $S^{n-1}$. Then, there exists a continuous function $f: B_{n} \rightarrow S^{k} \backslash\{\infty\}$ that tends to $\phi$ uniformly along radii ending in $E$ (in the sense of Bagemihl and McMillan) if and only if there is sequence of pairs $h_{m}: S^{n-1} \rightarrow S^{k} \backslash\{\infty\}$ and $\phi_{m}: S^{n-1} \rightarrow \mathcal{C}\left(S^{k}\right)(m=1,2, \ldots)$ of continuous functions such that each $h_{m}$ is a selection for $\phi_{m}$, the above condition (1) holds, and the sequence $\left\{\phi_{m}\right\}$ converges uniformly to $\phi$ on $E$. 
In a follow-up paper, we shall apply the above theorems to prove a longstanding conjecture of Bagemihl and Seidel [3, p. 99] along with some related results. The conjecture concerns the image sets $f^{\oplus}[E]$ of certain subsets $E$ of $S^{1}$ for functions $f$ that are analytic on the unit disk $D=B_{2}$. The related results deal with the "uncountable-order sets" for $f^{\oplus}$ (see [5] and [4] for discussions) as well as uniform convergence in the sense of Bagemihl and McMillan of $f$ to $f^{\oplus}$ along radii ending in a closed, nowhere dense set $E$. (See also [6].)

The paper is organized as follows. In Sections $2-4$ we develop the necessary material concerning Borel and Baire classes of functions, hyperspaces, and radial cluster set functions. The construction of the homotopies that are required for the proofs of Theorems 1.4 and 1.5 are carried out in Section 5. Finally, the proofs of the main theorems are begun in Section 3 and completed in Section 6.

We conclude the introduction with some conventions that will be followed in the paper. The overline notation for the closure $\bar{A}$ of a set $A$ has already been used in Theorem 1.4. The boundary and the diameter will be denoted by $\partial A$ and $\operatorname{diam}(A)$, respectively. The oscillation of a function $\phi$ on a set $A$, denoted $\operatorname{osc}_{A}(\phi)$, is $\operatorname{diam}(\phi[A])$. Finally, $I$ is the closed interval $[0,1]$ and $I^{\omega_{0}}$ is the Hilbert cube.

\section{Borel and Baire classes}

For the present section, $X$ and $Y$ are complete, separable metric spaces.

In the proofs of our interpolation theorems (Theorems 1.1 and 1.3), we will invoke measurable selection theorems. The selections will yield Borel measurable point-valued functions. The Borel measurable functions are classified into subclasses indexed by the countable ordinal numbers. This is analagous to the corresponding classification of Baire functions (the smallest class of functions that contains the continuous functions and is closed under pointwise convergence). We shall be concerned here only with the finite Borel and Baire classes. Even for the finite case, subclasses having the same index do not agree in general. (See [14, p. 391], [11], and [17].) We will prove in this section that the two classifications agree for mappings of $X$ into $S^{k}$ for $k \geq 1$.

Let us begin by recalling the definition of the finite Baire classes for functions $f: E \rightarrow Y$, where $E$ is a subspace of $X$. The inductive definition begins with the class $C(E, Y)$ of continuous functions from $E$ into $Y$.

Definition ([14, p. 392]). The function $f: E \rightarrow Y$ is of Baire class 0 precisely when $f \in C(E, Y)$. And, for a positive finite ordinal $\gamma$, the function $f$ is the said to be of Baire class $\gamma$ provided it is the pointwise limit of a sequence of functions contained in the Baire class $\gamma-1$.

Before proceeding to the definition of the Borel classes of functions, let us recall some facts about the Borel sets in the space $X$. The class of Borel sets is the smallest $\sigma$-algebra of sets containing the closed sets. The Borel sets are classified 
into subclasses indexed by the countable ordinal numbers (see [14, pp. 344-345]). In our discussion, we shall need only the three multiplicative classes $F_{0}, G_{1}, F_{2}$ and the three additive classes $G_{0}, F_{1}, G_{2}$ which are given below. (The multiplicative classes are closed under countable intersections and the additive classes are closed under countable unions.)

$$
\begin{array}{ll}
F_{0}=\{E: E \text { is a closed set }\}, & G_{0}=\{E: E \text { is an open set }\} \\
F_{1}=\left\{E: E \text { is an } F_{\sigma}-\text { set }\right\}, & G_{1}=\left\{E: E \text { is an } G_{\delta}-\text { set }\right\} \\
F_{2}=\left\{E: E \text { is an } F_{\sigma \delta}-\text { set }\right\}, & G_{2}=\left\{E: E \text { is an } G_{\delta \sigma}-\text { set }\right\} .
\end{array}
$$

For other finite ordinal numbers $\gamma$, the classes $F_{\gamma}$ and $G_{\gamma}$ are defined in the obvious way.

Definition ([14, p. 273]). For each finite ordinal number $\gamma$, a function $f: X \rightarrow Y$ is said to be of Borel class $\gamma$ if $f^{-1}[W]$ is a Borel set of multiplicative class $\gamma$ for each closed set $W$.

It is easy to verify for finite $\gamma$ that every function $f: X \rightarrow Y$ of Baire class $\gamma$ is also of Borel class $\gamma$. The converse is obviously false if one takes $X=R$ and the discrete space $Y=S^{0}=\{-1,1\}$. In this case, the class of Baire functions is precisely $C\left(R, S^{0}\right)$. The following classical theorem establishes the equivalence of the corresponding Borel and Baire classes for certain range spaces. (See [10, p. 143] and [14, p. 393].)

Theorem 2.1 (Lebesgue-Hausdorff). Let $\gamma$ be a positive integer. Then, the collection of Borel class $\gamma$ mappings of $X$ into $Y$ is the same as the collection of Baire class $\gamma$ mappings when $Y$ is $R^{k}$ or $I^{k}$ for $k=1,2, \ldots$, and when $Y$ is the Hilbert cube $I^{\omega_{0}}$.

Recently, C.A. Rogers [17] (see also [11]) gave sufficient conditions under which the Borel and Baire classes coincide, generalizing the classical Lebesgue-Hausdorff theorem. Unfortunately, Rogers' theorem does not yield the following analogue of the Lebesgue-Hausdorff theorem where $Y$ is the sphere $S^{k}$ for $k>0$.

Theorem 2.2. For positive integers $\gamma$ and $k$, a function $f: X \rightarrow S^{k}$ is of Baire class $\gamma$ if and only if it is of Borel class $\gamma$.

Proof. Necessity was noted before, so we shall concern ourselves only with the proof of sufficiency.

Suppose first that $\gamma=1$. Let $f: X \rightarrow S^{k}$ be of Borel class 1 . To prove that $f$ is of Baire class 1, we shall construct a sequence of continuous functions $\left\{f_{m}\right\}$ that converges pointwise to $f$. To this end, recall that $\infty=(0, \ldots, 0,1) \in S^{k} \subset R^{k+1}$ and define the sets

$$
A=f^{-1}[\{y \in Y:\|y-\infty\| \geq 1 / 4\}]
$$


and

$$
B=f^{-1}[\{y \in Y:\|y-\infty\| \leq 1 / 8\}] .
$$

Then the sets $A$ and $B$ are disjoint $G_{\delta}$-sets of $X$. By the separation theorem ([14, p. 350]), there is a subset $E$ of $X$ which is simultaneously an $F_{\sigma}$ and $G_{\delta}$-set such that $A \subset E$ and $B \subset X \backslash E$. Let $y_{0}$ be a point in $S^{k}$ with $\left\|y_{0}-\infty\right\|=1 / 8$ and define the functions $g_{1}$ and $g_{2}$ by

$$
g_{1}(x)= \begin{cases}f(x), & \text { if } x \in E \\ y_{0}, & \text { if } x \in X \backslash E\end{cases}
$$

and

$$
g_{2}=f-g_{1}
$$

Then,

$$
g_{1}: X \rightarrow\left\{y \in S^{k}:\|y-\infty\| \geq 1 / 8\right\}
$$

and

$$
g_{2}: X \rightarrow\left\{y \in R^{k+1}:\|y\| \leq 1 / 2\right\}
$$

are Borel class 1 functions. By the Lebesgue-Hausdorff theorem (Theorem 2.1), there are sequences of continuous functions

$$
g_{1 m}: X \rightarrow\left\{y \in S^{k}:\|y-\infty\| \geq 1 / 8\right\} \quad(m=1,2, \ldots)
$$

and

$$
g_{2 m}: X \rightarrow\left\{y \in R^{k+1}:\|y\| \leq 1 / 2\right\} \quad(m=1,2, \ldots)
$$

that converge pointwise to $g_{1}$ and $g_{2}$, respectively. Therefore, for each positive integer $m$, the function $h_{m}=g_{1 m}+g_{2 m}$ is a continuous mapping of $X$ into the set $\left\{y \in R^{k+1}: 1 / 2 \leq\|y\| \leq 3 / 2\right\}$ and the sequence $\left\{h_{m}\right\}$ converges pointwise to $g_{1}+g_{2}=f$. It follows, with $P$ denoting the radial projection of $R^{k+1} \backslash\{O\}$ onto $S^{k}$, that $f_{m}=P \circ h_{m}(m=1,2, \ldots)$ is a sequence of continuous functions into $S^{k}$ converging pointwise to $f$. This completes the proof that $f$ is of Baire class 1 from $X$ into $S^{k}$.

The case when $\gamma>1$ is handled by using the Banach theorem (see [14, p. 394]). This theorem asserts that for any positive integer $\gamma$, the class of pointwise limits of functions in the Borel class $\gamma$ is the Borel class $\gamma+1$.

Theorem 2.2 is thereby established.

Remark. As a consequence of Theorem 2.2, we also have that the functions $f: X \rightarrow S^{k} \times I^{j}$ and $f: X \rightarrow S^{k} \times S^{j}$ are of Baire class $\gamma$ when and only when they are of Borel class $\gamma$. An annular region in the plane and a 2 -dimensional torus are such spaces. Moreover, the above proof will work for any finitely connected Jordan region in the plane. 


\section{Hyperspaces and radial cluster set functions}

As in the introduction, we shall view radial cluster set functions $f^{\oplus}$ associated with continuous functions $f: B_{n} \rightarrow S^{k} \backslash\{\infty\}$ as mappings into certain hyperspaces. In the initial part of this section we define these spaces precisely and give some relevant results concerning them. The last part of the section will be devoted to properties of radial cluster set functions $f^{\oplus}$.

The collection of all nonempty, closed sets in $Y$ is denoted by $2^{Y}$. For the purposes of the present section, the space $Y$ will be assumed to be compact. The collection $\mathcal{C}(Y)$ will denote the subset of $2^{Y}$ consisting of the connected sets (that is, continua). The sets $2^{Y}$ and $\mathcal{C}(Y)$ possess a natural metric which we now define.

Definition. Suppose $Y$ is a compact space. The metric $d$, called the Hausdorff metric, is defined on $2^{Y}$ by

$$
d(K, F)=\max \{\operatorname{dist}(u, F), \operatorname{dist}(K, v): u \in K, v \in F\},
$$

for $K, F \in 2^{Y}$. The sets $2^{Y}$ and $\mathcal{C}(Y)$ endowed with the Hausdorff metric $d$ are called hyperspaces.

We infer from a theorem of D.W. Curtis and R.M. Shori [8, Theorem 2] and [9] the following fact.

Theorem 3.1. If $Y$ is any of the spaces $S^{k}$ or $I^{k}(k \geq 2)$, then $2^{Y}$ and $\mathcal{C}(Y)$ are homeomorphic to the Hilbert cube $I^{\omega_{0}}$.

The next corollary is an immediate consequence of the above theorem and the Lebesgue-Hausdorff theorem (Theorem 2.1).

Corollary 3.2. Let $\gamma$ be a positive integer and $Y$ be any of the spaces $S^{k}$ and $I^{k}(k \geq 2)$. Then, a function $f$ mapping a space $X$ into $2^{Y}$ or $\mathcal{C}(Y)$ is of Borel class $\gamma$ if and only if it is of Baire class $\gamma$.

We remark that the corollary is also true for $k=1$. This is established by employing the fact that $2^{S^{1}}$ and $2^{I}$ are homeomorphic to the Hilbert cube and that $\mathcal{C}\left(S^{1}\right)$ and $\mathcal{C}(I)$ are homeomorphic to $I^{2}$ (see [8] and [16], respectively).

When $k \geq 2$, the point $\infty$ is not a local cut point of $S^{k}$. That is, the set $N \backslash\{\infty\}$ is connected for each connected neighborhood $N$ of $\infty$. We shall call a member $K$ of $\mathcal{C}\left(S^{k}\right)$ an $\infty$-free continuum if it does not contain the point $\infty$. The following theorem appears in the first cited reference for the case $k=2$.

Theorem 3.3 ([6, p. 383], [15, pp. 49 and 260]). If $k \geq 2$, then the collection of $\infty$-free, locally connected continua in $S^{k}$ is dense in $\mathcal{C}\left(S^{k}\right)$.

The proof is elementary; and, the statement is false when $k=1$.

The locally connected continua are characterized as the continuous images of the unit interval $I$ (see the Hahn-Mazurkiewicz-Sierpinski theorem [15, p. 256]). Using this theorem, we obtain the following corollary of Theorem 3.3. 
Corollary 3.4. Let $k \geq 2$ and $\varepsilon>0$. If $K \in \mathcal{C}\left(S^{k}\right)$ and $y$ is a point of $S^{k}$ such that $\operatorname{dist}(y, K)<\varepsilon$ and $y \neq \infty$, then there exists a function $f$ in $C\left(I, S^{k} \backslash\{\infty\}\right)$ such that $f(0)=f(1)=y$ and $d(f[I], K)<\varepsilon$.

We shall end our discussion of hyperspaces with a short summary of the selection theorems needed in Section 6. As usual, a selection for a set-valued function $\phi: X \rightarrow 2^{Y}$ is a function $g: X \rightarrow Y$ such that $g(x) \in \phi(x)$ for each $x$ in $X$. The following theorem is a special case of a theorem of Himmelberg, Van Vleck, and Prikry [12, p. 128].

Theorem 3.5. Let $\gamma$ be any positive integer. If $\phi: X \rightarrow 2^{Y}$ is of Borel class $\gamma$, then $\phi$ has a Borel class $\gamma$ selection $g: X \rightarrow Y$.

(Note that in the original statement of the Himmelberg, Van Vleck, Prikry theorem, "Borel" is replaced by "Baire" since the authors defined their term "Baire" using our definition of "Borel".) Corollary 3.2 and Theorem 2.2 together with Theorem 3.5 yield the following corollary.

Corollary 3.6. Let $\gamma$ and $k$ be a positive integers. If $\phi$ is a Baire class $\gamma$ mapping of $X$ into $\mathcal{C}\left(S^{k}\right)$ or $2^{S^{k}}$, then $\phi$ has a Baire class $\gamma$ selection $g: X \rightarrow S^{k}$.

An immediate consequence of the Tietze Extension Theorem ([13, p. 82]) and Theorem 3.1 is the following.

Proposition 3.7. Let $k \geq 1$. If $E$ is an $F_{\sigma}$ subset of $X$ and $\phi$ is a Baire class 1 map of $E$ into $\mathcal{C}\left(S^{k}\right)$, then there is a sequence of continuous mappings $\phi_{m}: X \rightarrow \mathcal{C}\left(S^{k}\right)(m=1,2, \ldots)$ such that the sequence $\left\{\phi_{m}(x)\right\}$ converges to $\phi(x)$ for each $x$ in $E$.

In the upcoming lemmas, we shall need the notion of unstable values defined next.

Definition ([13, p. 74]). Let $f: X \rightarrow Y$ be a continuous map. A point $y$ of $Y$ is called an unstable value of $f$ if for each positive number $\varepsilon$ there is a continuous map $g: X \rightarrow Y$ such that dist $(f(x), g(x))<\varepsilon$ when $x \in X$ and such that $y \notin g[X]$. A point $y$ in $Y$ is called a stable value of $f$ when it is not unstable.

Concerning unstable values, the following useful lemma is found in [13, pp. $78-$ 79]. It will be used in Section 5 as well as in the proof of Lemma 3.9 below.

Lemma 3.8. Let $f: X \rightarrow R^{k}$ be a continuous function. Then, the origin $O$ of $R^{k}$ is an unstable value of $f$ if and only if for each positive number $\delta$ there is a continuous function $g: X \rightarrow R^{k}$ such that (i) $f(x)=g(x)$ for $\|f(x)\| \geq \delta$, (ii) $\|g(x)\| \leq \delta$ for $\|f(x)\| \leq \delta$, and (iii) $O \notin g[X]$.

The next lemma deals with the replacement of a double sequence of continuous functions having $\infty$ as an unstable value with a single finite-valued sequence of continuous functions. 
Lemma 3.9. Let $E$ be the union of a countable collection $\left\{E_{j}\right\}$ of closed subsets of $X$. Suppose that $g: E \rightarrow S^{k}$ and that $g_{j m}: E_{j} \rightarrow S^{k}$ is a continuous function having $\infty$ as an unstable value for each pair of positive integers $j$ and $m$. If

$$
\lim _{m \rightarrow \infty} g_{j m}(x)=g(x), \quad x \in E_{j} \quad(j=1,2, \ldots),
$$

then there is a sequence $g_{m}: X \rightarrow S^{k} \backslash\{\infty\}(m=1,2, \ldots)$ of continuous functions that converges pointwise to $g$ on $E$.

Proof. For each $m$, let

$$
F_{m}=\left\{y \in S^{k}:\|y-\infty\| \geq 1 / m\right\} .
$$

Since $E_{j}$ is closed, we infer from Lemma 3.8 and the Tietze Extension Theorem $[13$, p. 82$]$ that, for each $m$, there is a continuous function $h_{j m}: X \rightarrow F_{m}$ such that

$$
\left\|h_{j m}(x)-g_{j m}(x)\right\| \leq 3 / m, \quad x \in E_{j} \quad(j=1,2, \ldots) .
$$

For each pair of positive integers $m$ and $j$, let

$$
H_{j m}=\bigcap_{l<j}\left\{x \in E_{j}: \operatorname{dist}\left(x, E_{l}\right) \geq 1 / m\right\}
$$

Then, $H_{j m}$ is a closed set contained in $E_{j}$. And, for each $j$, the sequence $H_{j m}$ $(m=1,2, \ldots)$ is a nondescending sequence whose union is $E_{j} \backslash \cup_{l<j} E_{l}$. For each $m$, let $H_{m}$ be the closed set defined by $H_{m}=\cup_{j \leq m} H_{j m}$. Then, the sequence $\left\{H_{m}\right\}$ is a nondescending sequence whose union is $E$.

For each $m$, let $g_{m}(x)=h_{j m}(x)$ for $x \in H_{j m}$, where $j \leq m$. Then, $g_{m}$ is a continuous mapping of $H_{m}$ into $F_{m}$. By the Tietze Extension Theorem, the function $g_{m}$ has a continuous extension to a function mapping $X$ into $F_{m}$. Obviously, $\infty \notin g_{m}[X]$. A straightforward argument shows that $\left\{g_{m}\right\}$ converges pointwise to $g$ on $E$.

Using Lemma 3.9, we derive the following result relevant to Theorem 1.1.

Lemma 3.10. Let $E$ be an $F_{\sigma}$ subset of $X$ and let $\phi: E \rightarrow \mathcal{C}\left(S^{k}\right)$ be a Baire class 1 function such that $\phi(x)$ is $\infty$-free for each $x$ in $E$. Then, there is a sequence $h_{m}: X \rightarrow S^{k}(m=1,2, \ldots)$ of continuous functions such that each $h_{m}$ has $\infty$ as an unstable value and, for each $x$ in $E$, the sequence $\left\{h_{m}(x)\right\}$ converges to a point in $\phi(x)$.

Proof. We may assume that $E$ is the union of a nondescending sequence $\left\{E_{j}\right\}$ of closed subsets of $X$. Since $X$ is a complete metric space and $\left.\phi\right|_{E_{j}}$ is of Baire class 1 , by Corollary 3.6, there is a Baire class 1 selection $h_{j}: E_{j} \rightarrow S^{k}$ for $\left.\phi\right|_{E_{j}}$. By Theorem 2.2, the function $h_{j}$ is a Borel class 1 mapping of $E_{j}$ into $S^{k}$. 
Since $\phi(x)$ is $\infty$-free for each $x$ in $E$, we have that $h_{j}: E_{j} \rightarrow S^{k} \backslash\{\infty\}$. We shall show that $h_{j}$ is a Baire class 1 mapping of $E_{j}$ into $S^{k} \backslash\{\infty\}$. To this end, let $W$ be a relatively closed subset of $S^{k} \backslash\{\infty\}$. Then $W \cup\{\infty\}$ is closed in $S^{k}$. Hence, $h_{j}^{-1}[W]=h_{j}^{-1}[W \cup\{\infty\}]$ is a $G_{\delta}$-subset of $E_{j}$. We have shown that $h_{j}$ is a Borel class 1 mapping of $E_{j}$ into $S^{k} \backslash\{\infty\}$. Therefore, by the LebesgueHausdorff theorem (Theorem 2.1), the function $h_{j}$ is a Baire class 1 mapping of $E_{j}$ into $S^{k} \backslash\{\infty\}$. Consequently, for each $j$ and $m$ there is a continuous function $h_{j m}: E_{j} \rightarrow S^{k}$ such that $\lim _{m \rightarrow \infty} h_{j m}(x)=h_{j}(x)$ for each $x$ in $E_{j}$ and $h_{j m}$ has $\infty$ as an unstable value.

In order to apply Lemma 3.9, we must construct the function $g: E \rightarrow S^{k}$ along with the associated countably indexed collection of sets and the doubly indexed collection of functions. Here, we shall use a doubly indexed collection $\left\{E_{j m}\right\}$ of sets and a triply indexed collection $\left\{g_{j m l}\right\}$ of functions instead. For each $j$, the set $E_{j} \backslash E_{j-1}$ (with $E_{0}=\emptyset$ ) is an $F_{\sigma}$-set. So, for each $j$, there is a sequence $\left\{E_{j m}\right\}$ of closed sets whose union is $E_{j} \backslash E_{j-1}$. We now define $g(x)=h_{j}(x)$ when $x \in E_{j} \backslash E_{j-1}$ and define $g_{j m l}(x)=h_{j l}(x)$ when $x \in E_{j m}$. Lemma 3.9 completes the proof.

We turn now to the radial cluster set functions $f^{\oplus}$ associated with continuous functions $f: B_{n} \rightarrow S^{k}$. We refer the reader to [7, Chapter 1] for a general background concerning cluster sets. We shall begin with a precise definition of the radial cluster set function. Throughout the remainder of this section, all functions $f$ will be from the class $C\left(B_{n}, S^{k}\right)$ of continuous mappings of the ball $B_{n}$ into the sphere $S^{k}$.

Definition. Let $f: B_{n} \rightarrow S^{k}$ be a continuous function on the ball. For each point $\zeta$ of the boundary $S^{n-1}$ of $B_{n}$, let $f^{\oplus}(\zeta)$ denote the set of limit points of the convergent sequences $\left\{f\left(r_{m} \zeta\right)\right\}$, where $\left\{r_{m}\right\}$ is an increasing sequence of positive numbers converging to 1 . The function $f^{\oplus}$ is called the radial cluster set function associated with $f$.

Clearly, for $\zeta$ in $S^{n-1}$, we have

$$
\begin{aligned}
f^{\oplus}(\zeta) & =\bigcap_{0 \leq R<1} \overline{U_{R \leq S<1} f[\{r \zeta: R \leq r \leq S\}]} \\
& =\bigcap_{0 \leq R<1} \overline{f[\{r \zeta: R \leq r<1\}]} .
\end{aligned}
$$

Consequently, one can easily verify that $f^{\oplus}(\zeta)$ is in $\mathcal{C}\left(S^{k}\right)$ for each $\zeta$ in $S^{n-1}$. Moreover, we have from formula (3) above the following bound on the Baire class of $f^{\oplus}$.

Proposition 3.11. For each function $f$ in $C\left(B_{n}, S^{k}\right)$, the radial cluster set function $f^{\oplus}$ is of Baire class 2. 
The following example shows that the above proposition cannot be sharpened.

Example. Let $E$ and $H$ be mutually disjoint countable, dense subsets of $S^{1}$. By a theorem of Bagemihl and Seidel [2, p. 194], there exists an analytic function $f$ on $B_{2}$ such that

$$
f^{\oplus}(\zeta)= \begin{cases}\{1\}, & \zeta \in E \\ \{0\}, & \zeta \in H .\end{cases}
$$

As usual, we identify the complex plane $C$ with $R^{2}$; and, we have $\Pi^{-1}\left[R^{2}\right]=$ $S^{2} \backslash\{\infty\}$. Thus, we shall assume that the above function $f$ has values in $S^{2} \backslash\{\infty\}$. Consider the continuous function $g: \mathcal{C}\left(S^{2}\right) \rightarrow R$ given by

$$
g(W)=d(W,\{0\}), \quad W \in \mathcal{C}\left(S^{2}\right) .
$$

An easy calculation shows that $g \circ f^{\oplus}$ is discontinuous at each point of $S^{1}$. By a theorem of Baire (see [14, p. 419]), the function $g \circ f^{\oplus}$ is not of Borel class 1; consequently, $f^{\oplus}$ is also not of Baire class 1 .

Clearly, if $E$ is a subspace of $S^{n-1}$ and $f^{\oplus}(\zeta)$ is a singleton for each $\zeta$ in $E$, then $f^{\oplus}$ can be identified with a radial limit function on $E$. Such functions are necessarily of Baire class 1 . We state this fact as a proposition.

Proposition 3.12. Let $f$ be in $C\left(B_{n}, S^{k}\right)$. If $E$ is a subset of $S^{n-1}$ such that $\left.f^{\oplus}\right|_{E}$ is singleton-valued, then $\left.f^{\oplus}\right|_{E}$ is of Baire class 1 .

In [1, Definition 1], Bagemihl and McMillan defined a notion of uniform convergence of $f$ to $f^{\oplus}$. (Although this concept was originally defined for rotates of more general sets than radii, we shall, for simplicity, give only the definition for the radial case.)

Definition. Let $E$ be a subset of the boundary $S^{n-1}$ of $B_{n}$ and $f$ be in $C\left(B_{n}, S^{k}\right)$. Then, $f$ is said to tend or converge uniformly to $f^{\oplus}$ along the radii ending in $E$ provided the following holds: For each positive number $\varepsilon$ there exists a number $\delta_{\varepsilon}$ in $(0,1]$ such that for each $\delta$ in $\left(0, \delta_{\varepsilon}\right.$ ] there exists a $\lambda_{\delta}$ in $(0, \delta]$ such that if $\lambda \in\left(0, \lambda_{\delta}\right]$, then

$$
d\left(\{f(r \zeta): 1-\delta \leq r \leq 1-\lambda\}, f^{\oplus}(\zeta)\right)<\varepsilon, \quad \zeta \in E .
$$

The above definition is equivalent to the requirement that the following two conditions hold simultaneously:

(4) For each number $r$ in $[0,1)$, the family of real-valued functions

$$
d(f[[r \zeta, s \zeta]], \overline{f[[r \zeta, \zeta)]}), \quad \zeta \in E,
$$

converges uniformly to 0 as $s \rightarrow 1^{-}$. 
(5) The family of real-valued functions

$$
\left.d \overline{f[[r \zeta, \zeta)]}, f^{\oplus}(\zeta)\right), \quad \zeta \in E
$$

converges uniformly to 0 as $r \rightarrow 1^{-}$.

Let us show that if (4) and (5) hold simultaneously for the set $E$ then they also hold simultaneously for the closure $\bar{E}$ of the set $E$. Let $\varepsilon>0$ and $r \in[0,1)$. Then there is a positive number $\bar{s}$ such that $\bar{s} \leq s \leq s^{\prime}<1$ implies

$$
d\left(f[[r \zeta, s \zeta]], f\left[\left[r \zeta, s^{\prime} \zeta\right]\right]\right) \leq \varepsilon, \quad \zeta \in E
$$

The continuity of $f$ yields the same inequality for all $\zeta$ in $\bar{E}$. Letting $s^{\prime} \rightarrow 1^{-}$, we have

$$
d(f[[r \zeta, s \zeta]], \overline{f[[r \zeta, \zeta)]}) \leq \varepsilon, \quad \zeta \in \bar{E}
$$

when $\bar{s}<s<1$. Therefore, (4) holds for $\bar{E}$. We now conclude that $\overline{f[[r \zeta, \zeta)]}$ on $\bar{E}$ is continuous for each $r$ in $[0,1)$. By an analogous argument, if (5) also holds for $E$ then it holds for the closure $\bar{E}$; moreover, $f^{\oplus}$ restricted to $\bar{E}$ is continuous.

From the above discussion of the radial cluster set function $f^{\oplus}$, we shall find that formulas (3), (4) and (5) will lead us to the following set-valued function statements:

Consider a continuous function $f: B_{n} \rightarrow S^{k}$ and its associated radial cluster set function $f^{\oplus}: S^{n-1} \rightarrow \mathcal{C}\left(S^{k}\right)$. Suppose that $E$ is a subset of $S^{n-1}$ on which $f$ tends uniformly to $f^{\oplus}$ along radii ending in $E$. (Such a set always exists since any singleton subset of $S^{n-1}$ is such a set.) For the set $E$, we have from (4) and (5) the existence of an increasing sequence $\left\{r_{j}\right\}$ of positive numbers converging to 1 such that if $\phi_{j}$ is defined on $S^{n-1}$ by

$$
\phi_{j}(\zeta)=\left\{f(r \zeta): r_{j} \leq r \leq r_{j+1}\right\}, \quad \zeta \in S^{n-1},
$$

then $\left\{\phi_{j}\right\}$ converges to $f^{\oplus}$ uniformly on $E$ in the Hausdorff metric as $j$ tends to $\infty$. Associated with this continuous function $\phi_{j}$ on $S^{n-1}$ is a continuous point-valued function $h_{j}: S^{n-1} \rightarrow S^{k}$ given by

$$
h_{j}(\zeta)=f\left(r_{j} \zeta\right), \quad \zeta \in S^{n-1}
$$

Both $h_{j}$ and $h_{j+1}$ are selections for $\phi_{j}$. There appears to be no other natural connection between $h_{j}$ and $h_{j+1}$ which will yield necessary and sufficient conditions on the sequence $\left\{\phi_{j}\right\}$ of continuous functions of $S^{n-1}$ into $\mathcal{C}\left(S^{k}\right)$ so that a continuous function $f: B_{n} \rightarrow S^{k}$ for which $f^{\oplus}$ is the topological limit superior of the sequence $\left\{\phi_{j}\right\}$ will exist. (The definition of topological limit superior was given 
earlier in the introductory section, Section 1.) To establish another connection between $h_{j}$ and $h_{j+1}$, we shall exhibit another sequence $\left\{s_{m}\right\}$ of positive numbers increasing to 1 related to the sequence $\left\{r_{j}\right\}$. We infer from the compactness of the set

$$
\left\{x \in B_{n}: r_{j} \leq\|x\| \leq r_{j+1}\right\}
$$

the existence of numbers $t_{j, l}(l=0,1, \ldots, N(j))$ such that

$$
r_{j}=t_{j, 0}<t_{j, 1}<\cdots<t_{j, N(j)}=r_{j+1},
$$

and

$$
\max \left\{\left\|f\left(t_{j, l} \zeta\right)-f\left(t_{j, l-1} \zeta\right)\right\|: \zeta \in S^{n-1}\right\} \leq 1 / j, \quad(l=1, \ldots, N(j)) .
$$

Let $\left\{s_{m}\right\}$ be an increasing enumeration of the set

$$
\left\{t_{j, l}: j \geq 1 \text { and } 0 \leq l \leq N(j)\right\} \text {. }
$$

For convenience, let us repeat $\phi_{j}$ for each $m$ with $r_{j} \leq s_{m}<r_{j+1}$. Thus, we have the existence of a pair of continuous functions $h_{m}$ and $\phi_{m}$ defined by $h_{m}(\zeta)=f\left(s_{m} \zeta\right)$ and $\phi_{m}(\zeta)=\left\{f(r \zeta): r_{j} \leq r \leq r_{j+1}\right\}$ when $r_{j} \leq s_{m}<r_{j+1}$ and $\zeta \in S^{n-1}$. Clearly, $h_{m}$ is a selection for $\phi_{m}$, the sequence $\left\{\phi_{m}\right\}$ converges uniformly to $f^{\oplus}$ on the set $E, f^{\oplus}$ is the topological limit superior of the sequence $\left\{\phi_{m}\right\}$, and

$$
\lim _{m \rightarrow \infty} \max \left\{\left\|h_{m}(\zeta)-h_{m+1}(\zeta)\right\|: \zeta \in S^{n-1}\right\}=0 .
$$

We shall collect these facts into our next theorem. This theorem contains statement $(A)$ of Theorem 1.4 and the necessity part of Theorem 1.5.

Theorem 3.13. For $k \geq 1$, let $f: B_{n} \rightarrow S^{k}$ be a continuous function on the unit ball and $f^{\oplus}: S^{n-1} \rightarrow \mathcal{C}\left(S^{k}\right)$ be its associated radial cluster set function defined on the boundary $S^{n-1}$. Then, for each positive integer $m$, there is a pair of continuous functions $h_{m}$ and $\phi_{m}$ in $C\left(S^{n-1}, S^{k}\right)$ and $C\left(S^{n-1}, \mathcal{C}\left(S^{k}\right)\right)$ respectively such that

(i) $f^{\oplus}$ is the topological limit superior of the sequence $\left\{\phi_{m}\right\}$,

(ii) $h_{m}$ is a selection for $\phi_{m}$, and

(iii) $\lim _{m \rightarrow \infty} \max \left\{\left\|h_{m}(\zeta)-h_{m+1}(\zeta)\right\|: \zeta \in S^{n-1}\right\}=0$.

If $f$ is finite-valued (that is, $f(x) \neq \infty$ for all $x$ in $B_{n}$ ), then one can ensure that $\phi_{m}(\zeta)$ is $\infty$-free and $h_{m}(\zeta) \neq \infty$ for all $\zeta$ in $S^{n-1}$, and

$(\text { iii })^{\prime} \lim _{m \rightarrow \infty} \max \left\{\left\|\Pi \circ h_{m}(\zeta)-\Pi \circ h_{m+1}(\zeta)\right\|: \zeta \in S^{n-1}\right\}=0$.

Moreover, if $f$ tends uniformly to $f^{\oplus}$ along radii ending in a subset $E$ of $S^{n-1}$, then one can also ensure that the sequence $\left\{\phi_{m}\right\}$ converges uniformly to $f^{\oplus}$ on $E$.

Observe that $\phi_{m}(\zeta)$ can be taken to be the singleton $\left\{h_{m}(\zeta)\right\}$ for each $m$ and each $\zeta$ in $S^{n-1}$ in the discussion just preceding the statement of the theorem. With this observation, we note that the idea of this proof is similar to that appearing in [5, Lemmas 2.3 and 2.4]. 


\section{Triangulation and dimension}

We shall use triangulations of spaces to construct our homotopies in Section 5. Also, we shall use the connection between the dimension of a space and the unstable values of continuous maps. In this section, short discussions of triangulations and dimension will be given for the convenience of the reader.

Let us begin with a brief discussion of finitely triangulable spaces $X$, simplicial complexes $(K, S)$ and the barycenter of a simplex.

By an $m$-simplex $\sigma$, we shall mean the convex hull of the $m+1$ element set $\left\{x_{0}, \ldots, x_{m}\right\}$ in $R^{n}$, where the vectors $x_{i}-x_{0}(i=1, \ldots, m)$ are linearly independent. The points $x_{i}$ are called the vertices of the simplex $\sigma$; and, the point $b(\sigma)=\left(\sum_{i=0}^{m} x_{i}\right) /(m+1)$ is called the barycenter of the simplex $\sigma$. The simplex $\sigma$ will have lower dimensional simplices formed by the subsets of the set of vertices of $\sigma$. These simplices are called faces of the simplex $\sigma$.

The ordered pair $(K, S)$ consisting of a space $K$ along with a finite collection $S=\left\{\sigma_{1}, \ldots, \sigma_{j}\right\}$ of simplices in $R^{n}$ is said to be a simplicial complex provided the following conditions are satisfied.

(i) $K=\cup_{i=1}^{j} \sigma_{i}$.

(ii) If $\sigma \in S$ and $\tau$ is a face of $\sigma$, then $\tau \in S$.

(iii) If $\sigma, \tau \in S$, then either $\sigma \cap \tau$ is empty or else is a common face of $\sigma$ and $\tau$.

(As is customary, we shall sometimes suppress reference to the collection $S$ and just refer to a simplicial complex $K$.) The $m$-skeleton $\left(K_{m}, S_{m}\right)$ of a simplicial complex $(K, S)$ is the simplicial complex for which $S_{m}=\{\sigma \in S: \operatorname{dim} \sigma \leq m\}$. Finally, it is intuitively obvious that $\operatorname{dim} K=\max \{\operatorname{dim} \sigma: \sigma \in S\}$.

A space $X$ is said to be finitely triangulable if it is homeomorphic to a simplicial complex $K$. A triangulation of $X$ is a homeomorphism $T: K \rightarrow X$ and the mesh of the triangulation is the maximum of the numbers $\operatorname{diam}(T[\sigma]), \sigma \in S$. The following is an elementary result.

Theorem 4.1. If $X$ is a finitely triangulable space and $\varepsilon>0$, then there is a triangulation such that its mesh is less than $\varepsilon$.

For ease of exposition, we make the following definition.

Definition. Let $(K, S)$ be a simplicial complex and $Y$ be a space. For $f$ in $C(K, Y)$ we define the oscillation of $f$ with respect to $S$ to be the number

$$
\omega_{S}(f)=\max \left\{\operatorname{osc}_{\sigma}(f): \sigma \in S\right\} .
$$

Let us turn now to dimension. The theory of dimension for separable, metrizable spaces is well developed and can be found in [13]. We shall be dealing with spaces $X$ whose $\operatorname{dimensions} \operatorname{dim} X$ are intuitively obvious. The following theorem gives a characterization. 
Theorem 4.2 ([13, p. 83]). For each nonnegative integer $n$, a space $X$ has $\operatorname{dim} X \leq n$ if and only if for each closed set $F$ of $X$ and each continuous function $f$ from $F$ into $S^{n}$ there is a continuous function $g$ from $X$ into $S^{n}$ such that $\left.g\right|_{F}=f$.

In Section 3 we gave the definition of an unstable value. The following shows a connection between dimension and unstable values (see $[13$, p. 80]) which will be useful in Section 6.

Theorem 4.3. Let $X$ be a space with $\operatorname{dim} X<k, Y$ be a space containing an open set $G$ homeomorphic to $R^{k}$ and $f$ be a continuous mapping of $X$ into a space $Y$. Then, all values of $f$ contained in $G$ are unstable. Indeed, if $\varepsilon>0$ and $y \in G$, then there is a continuous mapping $g$ of $X$ into $Y$ such that the inequality $\operatorname{dist}(f(x), g(x))<\varepsilon$ holds for all $x$ in $X$, the equality $f(x)=g(x)$ holds for $x$ in $X \backslash f^{-1}[G]$, the value $g(x)$ is contained in $G$ for $x$ in $f^{-1}[G]$ and $y$ is not in $g[X]$.

\section{Interpolation by radial cluster set functions}

We shall need three types of continuous homotopies. The first corresponds to two continuous functions $g_{0}$ and $g_{1}$ from $X$ into $R^{k}$, the second to two continuous functions $h_{0}$ and $h_{1}$ from $X$ into $S^{k} \backslash\{\infty\}$, and the third to pairs of continuous functions $g: X \rightarrow R^{k}$ and $\phi: X \rightarrow \mathcal{C}\left(S^{k}\right)$.

Lemma 5.1. Suppose that $g_{0}$ and $g_{1}$ are continuous mappings of $X$ into $R^{k}$. Then, there is a homotopy $\mu: X \times I \longrightarrow R^{k}$ satisfying

$$
\mu(x, 0)=g_{0}(x) \text { and } \mu(x, 1)=g_{1}(x), \quad x \in X,
$$

such that $\nu=\Pi^{-1} \circ \mu$ satisfies

$$
\|\nu(x, s)-\nu(x, t)\| \leq M\left\|g_{0}(x)-g_{1}(x)\right\|
$$

for all $x$ in $X$ and all $s, t$ in $I$.

(Recall that $M$ is the Lipschitz constant corresponding to the inverse of the stereographic projection $\Pi: S^{k} \backslash\{\infty\} \rightarrow R^{k}$.)

Proof. Let $\mu(x, t)=(1-t) g_{0}(x)-t g_{1}(x)$ for $(x, t)$ in $X \times I$.

For the second type of homotopy, let $h_{0}$ and $h_{1}$ be continuous functions from $X$ into $S^{k} \backslash\{\infty\}$ and consider the closed set

$$
D=\left\{x \in X:\left\|h_{0}(x)-h_{1}(x)\right\| \leq 1\right\} .
$$

For each $x$ in $D$, there is a unique geodesic from $h_{0}(x)$ to $h_{1}(x)$ in $S^{k}$. Consequently, there is a natural homotopy

$$
\beta: D \times I \longrightarrow S^{k} \text {, }
$$

where $\beta(x, \cdot)$ is the geodesic from $h_{0}(x)$ to $h_{1}(x)$ for $x$ in $X$. Of course, $\beta$ need not have $\infty$ as an unstable value. We have the following lemma when $\infty$ is an unstable value of $\beta$. 
Lemma 5.2. Suppose that $\delta \in(0,1)$ and that $h_{0}$ and $h_{1}$ are continuous functions from $X$ into $F_{\delta}=\left\{y \in S^{k}:\|y-\infty\| \geq \delta\right\}$. Let $E$ be a closed subset of $X$ and let $D$ and $\beta: D \times I \longrightarrow S^{k}$ be defined as above. If $\left.\beta\right|_{(E \cap D) \times I}$ has $\infty$ as an unstable value, then for each positive number $\varepsilon$ there is a homotopy $\alpha: X \times I \longrightarrow S^{k} \backslash\{\infty\}$ such that

$$
\alpha(x, 0)=h_{0}(x) \quad \text { and } \quad \alpha(x, 1)=h_{1}(x), \quad x \in X,
$$

and

$$
\|\alpha(x, t)-\beta(x, t)\|<\varepsilon, \quad(x, t) \in(D \cap E) \times I .
$$

Proof. Without loss of generality, suppose $\varepsilon<\delta / 2$. We infer from Lemma 3.8 the existence of a continuous function

$$
\nu:(D \cap E) \times I \longrightarrow S^{k} \backslash\{\infty\}
$$

such that $\nu(x, 0)=\beta(x, 0)=h_{0}(x)$ and $\nu(x, 1)=\beta(x, 1)=h_{1}(x)$ for all $x$ in $D \cap E$, and

$$
\|\nu(x, t)-\beta(x, t)\|<\varepsilon / 2, \quad(x, t) \in(D \cap E) \times I .
$$

Next, we shall denote by $P$ the natural retraction of $S^{k} \backslash\{\infty\}$ onto the closed set $\left\{y \in S^{k}:\|y-\infty\| \geq \varepsilon / 2\right\}$ and let $\alpha=P \circ \nu$. Then,

$$
\alpha(x, 0)=h_{0}(x) \text { and } \alpha(x, 1)=h_{1}(x), \quad x \in D \cap E,
$$

and

$$
\|\alpha(x, t)-\beta(x, t)\|<\varepsilon / 2, \quad(x, t) \in(D \cap E) \times I .
$$

Finally, extend $\alpha$ to $(X \times\{0,1\}) \cup((D \cap E) \times I)$ by letting

$$
\alpha(x, 0)=h_{0}(x) \text { and } \alpha(x, 1)=h_{1}(x), \quad x \in X,
$$

and then apply the Tietze Extension Theorem [13, p. 82] to complete the proof.

We shall have need of the next lemma concerning $h_{0}$ and $h_{1}$.

Lemma 5.3. Let $h_{0}$ and $h_{1}$ be continuous mappings of $X$ into $S^{k} \backslash\{\infty\}$ such that $\left\|h_{0}(x)-h_{1}(x)\right\| \leq 1$ for $x$ in $X$. If

$$
\left\|h_{0}(x)-h_{1}(x)\right\|<\left\|h_{0}(x)-\infty\right\|, \quad x \in X,
$$

then there is a homotopy $\alpha: X \times I \longrightarrow S^{k} \backslash\{\infty\}$ such that

$$
\alpha(x, 0)=h_{0}(x) \quad \text { and } \quad \alpha(x, 1)=h_{1}(x), \quad x \in X,
$$

and

$$
\operatorname{diam}(\alpha(x, I))=\left\|h_{0}(x)-h_{1}(x)\right\|, \quad x \in X .
$$

Proof. Obviously, the unique geodesic joining $h_{0}(x)$ and $h_{1}(x)$ in $S^{k}$ does not contain $\infty$. Consequently, there is a natural way to construct the homotopy $\alpha$.

In order to construct the third homotopy mentioned at the beginning of this section, we will need some auxiliary propositions. 
Proposition 5.4. Let $h: X \rightarrow S^{k}$ and $\psi: X \rightarrow 2^{S^{k}}$ be continuous functions. Then, there is a continuous function $\phi: X \rightarrow 2^{S^{k}}$ such that, for each $x$ in $X$,

$$
\psi(x) \subset \phi(x), \quad h(x) \in \phi(x)
$$

and

$$
d(\phi(x), \psi(x)) \leq 2 \operatorname{dist}(h(x), \psi(x)) .
$$

If, for some $x, \psi(x)$ is in $\mathcal{C}\left(S^{k}\right)$, then $\phi(x)$ can be ensured to be in $\mathcal{C}\left(S^{k}\right)$ also.

Proof. Let $\rho: X \rightarrow R$ be the continuous function defined by

$$
\rho(x)=\operatorname{dist}(h(x), \psi(x)), \quad x \in X,
$$

and put

$$
\phi(x)=\psi(x) \cup\left\{y \in S^{k}:\|y-h(x)\| \leq \rho(x)\right\} .
$$

One easily verifies that $\phi$ satisfies the conditions of the proposition.

Proposition 5.5. There exists a continuous function

$$
\Phi: C\left(I, R^{k}\right) \times C\left(I, R^{k}\right) \times I \times I \longrightarrow R^{k}
$$

such that

(i) $\Phi(f, g, 0, I)=f[I]$ and

$f[I] \subset \Phi(f, g, s, I) \subset f[I] \cup g[I] \cup[f(1), g(0)]$ for $s$ in $[0,1 / 2]$,

(ii) $\Phi(f, g, 1, I)=g[I]$ and and

$g[I] \subset \Phi(f, g, s, I) \subset f[I] \cup g[I] \cup[f(1), g(0)]$ for $s$ in $[1 / 2,1]$,

(iii) $\Phi(f, g, s, 0)=\Phi(f, g, s, 1)=h(s) \equiv(1-s) f(0)+s g(1)$ for $s$ in $I$.

Proof. We shall construct a continuous function

$$
\Lambda: C\left(I, R^{k}\right) \times C\left(I, R^{k}\right) \times[0,4] \times[-6,6] \longrightarrow R^{k}
$$

which, when rescaled, will result in the desired function $\Phi$. We will define $\Lambda$ on the subset of the domain defined with $[-6,6]$ replaced by $[0,6]$. The function is then extended to the entire domain by the symmetry condition

$$
\Lambda(f, g, s, t)=\Lambda(f, g, s,-t) .
$$

We first define two continuous functions

$$
\phi: C\left(I, R^{k}\right) \times C\left(I, R^{k}\right) \times[0,7] \longrightarrow R^{k}
$$


and

$$
\psi: C\left(I, R^{k}\right) \times C\left(I, R^{k}\right) \times[0,4] \longrightarrow R^{k}
$$

as follows:

$$
\phi(f, g, u)= \begin{cases}f(1), & 0 \leq u \leq 2 \\ f(3-u), & 2 \leq u \leq 3 \\ (4-u) f(0)+(u-3) g(1), & 3 \leq u \leq 4 \\ g(5-u), & 4 \leq u \leq 5 \\ g(0), & 5 \leq u \leq 7\end{cases}
$$

and

$$
\psi(f, g, v)= \begin{cases}(1-v) f(0)+v g(1), & 0 \leq v \leq 1 \\ g(2-v), & 1 \leq v \leq 2 \\ g(0), & 2 \leq v \leq 4\end{cases}
$$

We define $\Lambda$ on the set $[0,4] \times[0,6]$ by

$$
\Lambda(f, g, s, t)=\left\{\begin{array}{lll}
\phi(f, g, s+t), & (s, t) \in[0,4] \times[0,3] & \\
\psi(f, g, 3-t+s), & (s, t) \in[0,4] \times[3,6], & t-s \leq 2 \\
\psi(f, g, s /(t-2)), & (s, t) \in[0,4] \times[3,6], & t-s \geq 2 .
\end{array}\right.
$$

Figure 1 below depicts graphically some of the significant features of the function $\Lambda$.

It is a routine matter to prove that $\Lambda$ is continuous. The following facts are also easily checked.

(i) $f[I]=\Lambda(f, g, 0,[-6,6])$ and

$f[I] \subset \Lambda(f, g, s,[-6,6]) \subset f[I] \cup g[I] \cup[f(1), g(0)]$ for $s$ in $[0,2]$,

(ii) $g[I]=\Lambda(f, g, 1,[-6,6])$ and and$$
g[I] \subset \Lambda(f, g, s,[-6,6]) \subset f[I] \cup g[I] \cup[f(1), g(0)] \text { for } s \text { in }[2,4],
$$

(iii) $\Lambda(f, g, s,-6)=\Lambda(f, g, s, 6)=\frac{1}{4}(4-s) f(0)+\frac{1}{4} s g(1)$ for $s$ in $[0,4]$.

The rescaling of $\Lambda$ to obtain $\Phi$ presents no difficulty. This completes the proof of the proposition.

It is to be noted that the function $\Phi$ of Proposition 5.5 is not commutative in the first two variables, that is, we do not necessarily have $\Phi(f, g, s, t)=\Phi(g, f, s, t)$. Nor, is it true that $f(\cdot)=\Phi(f, g, 0, \cdot)$ and $g(\cdot)=\Phi(f, g, 1, \cdot)$.

Our final homotopy concerns functions $g: X \rightarrow R^{k}$ and $\phi: X \rightarrow \mathcal{C}\left(S^{k}\right)$. Let us begin with the existence of certain homotopies for simplicial complexes.

Lemma 5.6. Suppose that $(K, S)$ is a simplicial complex and that the functions $\psi$ in $C\left(K, \mathcal{C}\left(S^{k}\right)\right)$ and $g$ in $C\left(K, R^{k}\right)$ are such that $h=\Pi^{-1} \circ g$ is a selection for $\psi$, where $k \geq 2$. Let $\varepsilon$ be a positive number such that

$$
\omega_{S}(\psi)<\varepsilon \text { and } \omega_{S}(g)<\varepsilon .
$$




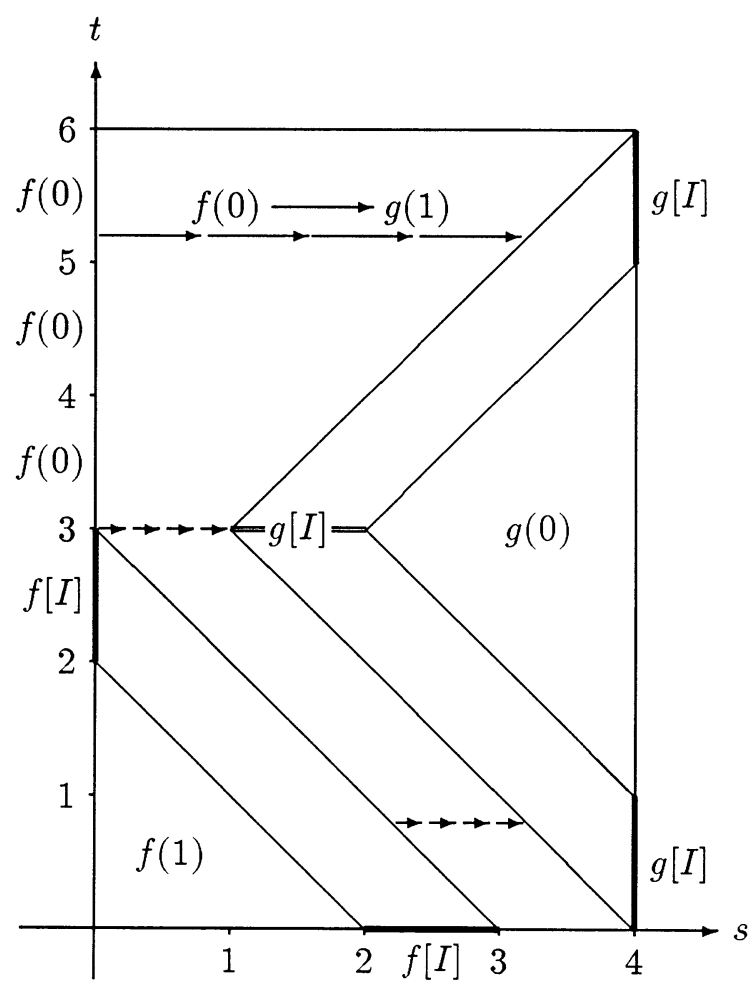

Figure 1.

Then, there is a function $\alpha$ in $C\left(K \times I, R^{k}\right)$ satisfying

$$
\alpha(x, 0)=\alpha(x, 1) \text { and }\|\alpha(x, 0)-g(x)\| \leq 5^{\operatorname{dim} K} \varepsilon, \quad x \in K,
$$

such that $\beta=\Pi^{-1} \circ \alpha$ satisfies

$$
d(\psi(x), \beta(x, I)) \leq 5^{\operatorname{dim} K} M \varepsilon, \quad x \in K .
$$

Proof. The proof is by induction on $\operatorname{dim} K$. When $\operatorname{dim} K=0$, the space $K$ is a finite set. So, the existence of the function $\alpha$ with the required properties follows immediately from Corollary 3.4 when $\operatorname{dim} K=0$.

Suppose now that $n$ is a positive integer, $\operatorname{dim} K=n$, and that the proposition holds for simplicial complexes having dimension less than $n$. Recall that $b(\sigma)$ denotes the barycenter of a simplex $\sigma$ and that $\left(K_{n-1}, S_{n-1}\right)$ denotes the $(n-1)$ skeleton of $(K, S)$. Clearly, $(\widetilde{K}, \widetilde{S})$ given by

$$
\widetilde{S}=S_{n-1} \cup\{\{b(\sigma)\}: \sigma \in S, \operatorname{dim} \sigma=n\}
$$


and

$$
\widetilde{K}=\cup \widetilde{S}=K_{n-1} \cup\{b(\sigma): \sigma \in S, \operatorname{dim} \sigma=n\}
$$

is a simplicial complex with $\operatorname{dim} \widetilde{K}=n-1$. Observe that the general assumptions on the functions $\psi, g$, and $h$ apply to their restrictions to $\widetilde{K}$. By the induction hypothesis, there exists a function $\widetilde{\alpha}$ in $C\left(\widetilde{K} \times I, R^{k}\right)$ satisfying the conclusions of the proposition with $\operatorname{dim} K$ replaced by $\operatorname{dim} \widetilde{K}=n-1$.

The construction of the function $\alpha$ will be made in two steps. We first define $\alpha$ on $\widetilde{K}$ as follows:

$$
\alpha(\zeta, t)=\Phi(\widetilde{\alpha}(\zeta, \cdot), \widetilde{\alpha}(\zeta, \cdot), 0, t), \quad(\zeta, t) \in K_{n-1} \times I,
$$

and, for each $\sigma$ in $S$ with $\operatorname{dim} \sigma=n$,

$$
\alpha(\zeta, t)=\Phi(\widetilde{\alpha}(\zeta, \cdot), \widetilde{\alpha}(\zeta, \cdot), 1, t), \quad \zeta=b(\sigma), \quad t \in I,
$$

where $\widetilde{\alpha}(\zeta, \cdot)$ denotes the restriction of $\widetilde{\alpha}$ to $\{\zeta\} \times I$ and $\Phi$ is the function defined in Proposition 5.5. For the second step of the construction, we will extend the function $\alpha$ to each $n$-simplex $\sigma$ in $S$. Let $\sigma$ be one such $n$-simplex. Since the boundary $\partial \sigma$ as well as the barycenter $b(\sigma)$ are contained in $\widetilde{K}$, we shall use the the function $\Phi$ to construct the desired extension. For each $x$ not in $\partial \sigma \cup\{b(\sigma)\}$, let $\zeta=\zeta(x)$ be the unique point of $\partial \sigma$ such that the line segment $\mu_{\zeta} \equiv[\zeta, b(\sigma)]$ contains $x$ and then let $p_{\zeta}: \mu_{\zeta} \rightarrow I$ be the linear function such that $p_{\zeta}(\zeta)=0$ and $p_{\zeta}(b(\sigma))=1$. Then, we extend $\alpha$ to $\sigma$ as follows:

$$
\alpha(x, t)=\Phi\left(\widetilde{\alpha}(\zeta, \cdot), \widetilde{\alpha}(b(\sigma), \cdot), p_{\zeta}(x), t\right), \quad(x, t) \in \sigma \times I .
$$

Now, the construction of the function $\alpha: K \times I \longrightarrow R^{k}$ is completed. Clearly, $\alpha$ is continuous.

Let us verify that $\alpha$ has the required properties. If $x$ is in $\widetilde{K}$, the result follows from the induction hypothesis and the properties of the function $\Phi$ of Proposition 5.5. So, we assume that $x$ is in some $n$-simplex $\sigma$ of $S$, indeed, in $\sigma \backslash(\partial \sigma \cup\{b(\sigma)\})$. The condition (iii) of Propositon 5.5 ensures that $\alpha(x, 0)=$ $\alpha(x, 1)$. To show that $\|\alpha(x, 0)-g(x)\| \leq 5^{\operatorname{dim} K} \varepsilon$, let $\zeta=\zeta(x)$ be as in the construction of $\alpha(x, t)$. Using the induction hypothesis and the condition on the oscillation of $g$, we obtain

$$
\begin{aligned}
\|\alpha(\zeta, 0)-\alpha(b(\sigma), 0)\| \leq & \|\alpha(\zeta, 0)-g(\zeta)\|+\|g(\zeta)-g(b(\sigma))\| \\
& +\|g(b(\sigma))-\alpha(b(\sigma), 0)\| \\
& <\left(2 \cdot 5^{n-1}+1\right) \varepsilon
\end{aligned}
$$

so that

$$
\begin{aligned}
&\|g(x)-\alpha(x, 0)\| \leq\|g(x)-g(b(\sigma))\|+\|g(b(\sigma))-\alpha(b(\sigma), 0)\| \\
&+\|\alpha(b(\sigma), 0)-\alpha(\zeta, 0)\| \\
&<\varepsilon+5^{n-1} \varepsilon+\left(2 \cdot 5^{n-1}+1\right) \varepsilon<5^{n} \varepsilon
\end{aligned}
$$


as required.

Essentially the same calculation as above, with $\psi$ in place of $g$ and the Hausdorff metric $d$ in place of the Euclidean norm, proves that the condition $d(\psi(x), \beta(x, I)) \leq 5^{n} M \varepsilon$ also holds.

The proof of Lemma 5.6 is concluded.

We are now able to prove our third homotopy lemma.

Lemma 5.7. Let $X$ be a finitely triangulable space, $\varepsilon>0$ and $k \geq 2$. If $g: X \rightarrow R^{k}$ and $\phi: X \rightarrow \mathcal{C}\left(S^{k}\right)$ are continuous, then there is a homotopy $\alpha: X \times I \longrightarrow R^{k}$ such that, for all $x$ in $X$,

(i) $\alpha(x, 0)=\alpha(x, 1)$,

(ii) $\alpha(x, 0)=g(x)$,

and

(iii) $d\left(\Pi^{-1}[\alpha(x, I)], \phi(x)\right)<2 \operatorname{dist}\left(\Pi^{-1} \circ g(x), \phi(x)\right)+\varepsilon M$, where $\Pi$ is the stereographic projection of $S^{k} \backslash\{\infty\}$ onto $R^{k}$ and $M$ is the Lipschitz constant of $\Pi^{-1}$.

Proof. The proof is a simple combination of Proposition 5.4, Theorem 4.1, Lemma 5.6 and Lemma 5.1.

\section{Proofs of the main theorems}

This section is devoted to the proofs of our main theorems. The statement (A) of Theorem 1.4 and the necessity part of Theorem 1.5 have already been proved by Theorem 3.13. Let us first prove a lemma.

Lemma 6.1. Let $X$ be a finitely triangulable space and $k \geq 2$. Suppose that $g_{m}: X \rightarrow R^{k}$ and $\psi_{m}: X \rightarrow \mathcal{C}\left(S^{k}\right)(m=1,2, \ldots)$ is a sequence of pairs of continuous functions. Then there is a continuous function $F: X \times[0, \infty) \longrightarrow R^{k}$ such that the topological limit superior $\phi$ of the sequence $\left\{\psi_{m}\right\}$ satisfies

(i) $\phi(x) \subset \overline{\Pi^{-1}[F(x,[j, \infty))]}$ for all $x$ and all $j$ and

(ii) $d\left(\psi_{m}(x), \Pi^{-1}[F(x,[m-1, m])]\right)$ converges to 0 at each $x$ for which

$$
\left\|g_{m}(x)-g_{m+1}(x)\right\|+\operatorname{dist}\left(\Pi^{-1} \circ g_{m}(x), \psi_{m}(x)\right)
$$

converges to 0 as $m$ tends to $\infty$.

Moreover, if $E$ is a subset of $X$ on which the sequence $\left\{\psi_{m}\right\}$ converges uniformly and on which the sequence

$$
\left\{\left\|g_{m}(\cdot)-g_{m+1}(\cdot)\right\|+\operatorname{dist}\left(\Pi^{-1} \circ g_{m}(\cdot), \psi_{m}(\cdot)\right)\right\}
$$

converges uniformly to 0 , then the sequence

$$
\left\{d\left(\phi(\cdot), \Pi^{-1}[F(\cdot,[m-1, m])]\right)\right\}
$$

also converges uniformly to 0 on $E$. 
Proof. We shall construct $F$ on $X \times[m-1, m]$. Let $\phi_{m}$ be the continuous function given by Lemma 5.4 corresponding to the functions $h_{m}=\Pi^{-1} \circ g_{m}$ and $\psi_{m}$. Then, $h_{m}$ is a selection of $\phi_{m}$ and $\psi_{m}(x) \subset \phi_{m}(x)$ for each $x$ in $X$. Let $\lambda_{m}$ be the homotopy joining $g_{m}$ and $g_{m+1}$ given by Lemma 5.1, and let $\alpha_{m}$ be the homotopy corresponding to $g_{m}, \phi_{m}$ and $\varepsilon_{m}=1 / m$ given by Lemma 5.7. Now, define $F$ on $X \times[m-1, m]$ by using $\alpha_{m}$ on the interval $\left[m-1, m-\frac{1}{2}\right]$ and $\lambda_{m}$ on the interval $\left[m-\frac{1}{2}, m\right]$. One easily verifies that the function $F$ satisfies the requirements of the lemma.

Now the proofs of the remaining parts of Theorems 1.4 and 1.5 will follow easily. For the statement (B) of Theorem 1.4, one easily can construct a function

$$
f:\left\{x \in B_{n}: 1 / 2 \leq\|x\|<1\right\} \longrightarrow S^{k} \backslash\{\infty\}
$$

with the required radial limit behavior by applying Lemma 6.1. The Tietze Extension Theorem permits the extension of the function $f$ to all of $B_{n}$. The sufficiency part of Theorem 1.5 is equally easily established.

Proof of Theorem 1.1. Suppose $E$ is an $F_{\sigma}$ set in $S^{n-1}$ and $\phi: E \rightarrow \mathcal{C}\left(S^{k}\right)$ is a Baire class 1 function with $\infty \notin \phi(x)$ for each $x$ in $E$. Since $E$ is an $F_{\sigma}$ set, we have by Proposition 3.7 a sequence $\psi_{m}: S^{n-1} \rightarrow \mathcal{C}\left(S^{k}\right)(m=1,2, \ldots)$ of continuous functions such that

$$
\lim _{m \rightarrow \infty} \psi_{m}(x)=\phi(x), \quad x \in E .
$$

By Proposition 3.10, there is a sequence $h_{m}: S^{n-1} \rightarrow S^{k}(m=1,2, \ldots)$ of continuous functions such that $\infty$ is an unstable value of each $h_{m}$ and

$$
\lim _{m \rightarrow \infty} h_{m}(x) \in \phi(x), \quad x \in E .
$$

Indeed, we may assume that $h_{m}(x) \neq \infty$ for all $x$ and all $m$. For each $m$, let $g_{m}=\Pi \circ h_{m}$. Then,

$$
\lim _{m \rightarrow \infty}\left\|g_{m}(x)-g_{m+1}(x)\right\|=0, \quad x \in E,
$$

because $\lim _{m \rightarrow \infty} h_{m}(x)$ exists and is not equal to $\infty$. Lemma 6.1 will now complete the proof of Theorem 1.1 in the same manner as it concluded the proof of Theorem 1.4.

Proof of Theorem 1.2. Let $k \geq 1$ and suppose that $h: S^{k} \rightarrow S^{k}$ is a continuous function. Let $x_{m}=\left(0, \ldots, 0, a_{m}\right) \in R^{k}$ be determined by an increasing sequence $\left\{a_{m}\right\}$ of real numbers such that $\lim _{m \rightarrow \infty} a_{m}=\infty$ and $\lim _{m \rightarrow \infty}\left(a_{m+1}-a_{m}\right)=0$. Clearly, $\lim _{m \rightarrow \infty} \Pi^{-1}\left(x_{m}\right)=\infty$. For each $m$, let $h_{m}: S^{k} \rightarrow S^{k} \backslash\{\infty\}$ be a continuous function such that $h_{m}(x)=h(x)$ if $\|h(x)-\infty\| \geq 1 / m$ and such 
that $h_{m}(x)=\Pi^{-1}\left(x_{m}\right)$ if $h(x)=\infty$. Such a function will exist by the Tietze Extension Theorem. Obviously, the sequence $\left\{h_{m}\right\}$ converges pointwise to $h$. With $g_{m}=\Pi \circ h_{m}$, we have that

$$
\lim _{m \rightarrow \infty}\left\|g_{m}(x)-g_{m+1}(x)\right\|=0, \quad x \in S^{k-1} .
$$

Now, the continuous function $F: S^{k} \times[0, \infty) \longrightarrow S^{k} \backslash\{\infty\}$ can be constructed by using Lemma 5.1 on the functions $g_{m}$ and $g_{m+1}$ to define $F$ on $S^{k} \times[m-1, m]$ for all positive integers $m$. This completes the proof of Theorem 1.2.

The proof of Theorem 1.3 will follow from the next two lemmas.

Lemma 6.2. Let $X$ be a finitely triangulable space and $E$ be an $F_{\sigma}$ subset of $X$ such that $\operatorname{dim} E \leq k-2$, where $k \geq 2$. Suppose that $\psi_{m}: X \rightarrow \mathcal{C}\left(S^{k}\right)$ and $h_{m}: X \rightarrow S^{k} \backslash\{\infty\}(m=1,2, \ldots)$ is a sequence of pairs of continuous functions. Then, there is a continuous function $F: X \times[0, \infty) \longrightarrow S^{k} \backslash\{\infty\}$ such that the topological limit superior $\phi$ of the the sequence $\left\{\psi_{m}\right\}$ satisfies

(i) $\phi(x) \subset \overline{F(x,[j, \infty))}$ for all $x$ and all $j$, and

(ii) $d\left(\psi_{m}(x), F(x,[m-1, m])\right)(m=1,2, \ldots)$ converges to 0 whenever $\lim _{m \rightarrow \infty}\left(\left\|h_{m}(x)-h_{m+1}(x)\right\|+\operatorname{dist}\left(h_{m}(x), \psi_{m}(x)\right)\right)=0$.

Moreover, if $H$ is a closed set contained in $E$ such that $\left\{\psi_{m}\right\}$ converges uniformly on $H$ and the sequence $\left\{\left\|h_{m}-h_{m+1}\right\|+\operatorname{dist}\left(h_{m}, \psi_{m}\right)\right\}$ converges uniformly to 0 on $H$, then the sequence $\{d(\phi(\cdot), F(\cdot,[m-1, m]))\}$ also converges uniformly to 0 on $H$.

Proof. The proof is similar to that of Lemma 6.1. We first construct a sequence $\phi_{m}: X \rightarrow \mathcal{C}\left(S^{k}\right)(m=1,2, \ldots)$ of continuous functions by using Lemma 5.4 such that $h_{m}$ is a selection for $\phi_{m}$ and $\psi_{m}(x) \subset \phi_{m}(x)$ for all $x$ in $X$. We then use the same homotopy $\alpha_{m}$ as in the proof of Lemma 6.1. The proof will use a different homotopy joining $h_{m}$ and $h_{m+1}$. Let $\left\{E_{m}\right\}$ be an nondecreasing sequence of closed sets whose union is $E$. For each $m$, let

$$
D_{m}=\left\{x \in X:\left\|h_{m}(x)-h_{m+1}\right\| \leq 1\right\} .
$$

The set $\left(E_{m} \cap D_{m}\right) \times I$ has dimension less than $k$ by the product theorem of dimension theory [13, p. 33]. If $\beta_{m}:\left(E_{m} \cap D_{m}\right) \times I \longrightarrow S^{k}$ is the natural homotopy from $h_{m}$ to $h_{m+1}$ on $E_{m} \cap D_{m}$ defined by means of the unique geodesic joining $h_{m}(x)$ to $h_{m+1}(x)$, then $\beta_{m}$ has $\infty$ as an unstable value by Theorem 4.3. Let $\lambda_{m}: X \times I \longrightarrow S^{k} \backslash\{\infty\}$ be the homotopy corresponding to $h_{m}, h_{m+1}$ and $\varepsilon_{m}=$ $1 / m$ on $E_{m} \cap D_{m}$ given by Lemma 5.2. (Note that the compactness of $E_{m} \cap D_{m}$ yields the appropriate $\delta$ in Lemma 5.2.) The function $F$ is now constructed in an analogous manner as in Lemma 6.1 by using $\alpha_{m}$ and $\lambda_{m}$. The verification of the 
properties of $F$ is made by using the following observation: If a point $x$ of $E$ is such that

$$
\lim _{m \rightarrow \infty}\left\|h_{m}(x)-h_{m+1}(x)\right\|=0,
$$

then there is an $m_{0}$ such that $x$ is in $E_{m} \cap D_{m}$ when $m \geq m_{0}$.

Lemma 6.3. Let $X$ be a finitely triangulable space and $E$ be an $F_{\sigma}$ subset of $X$ such that $\operatorname{dim} E \leq k-2$, where $k \geq 2$. Then, for a Baire class 1 function $\phi: E \rightarrow \mathcal{C}\left(S^{k}\right)$, there is a continuous function $F: X \times[0, \infty) \longrightarrow S^{k} \backslash\{\infty\}$ such that

$$
\phi(x)=\bigcap_{1 \leq m} \overline{F(x,[m, \infty))}, \quad x \in E .
$$

Proof. By Proposition 3.7, there is a sequence $\psi_{m}: X \rightarrow \mathcal{C}\left(S^{k}\right)(m=1,2, \ldots)$ of continuous functions such that $\lim _{m \rightarrow \infty} \psi_{m}(x)=\phi(x)$ for each $x$ in $E$. Let $\left\{E_{m}\right\}$ be a nondecreasing sequence of closed sets whose union is $E$. Then, the Baire class 1 function $\left.\phi\right|_{E_{m}}$ has a selection $H_{m}: E_{m} \rightarrow S^{k}$ of Baire class 1 (Corollary 3.6). Since $E_{m} \subset E_{m+1}$, we may assume that $H_{m}(X)=H_{m+1}(x)$ when $x$ is in $E_{m}$. Let $h: E \rightarrow S^{k}$ be defined by $h(x)=H_{m}(x)$ for $x$ in $E_{m}$. For each $m$ and each $j$, there is a continuous function $h_{j m}: E_{m} \rightarrow S^{k}$ such that $\lim _{j \rightarrow \infty} h_{j m}(x)=h(x)$ for each $x$ in $E_{m}$. Since $\operatorname{dim} E_{m}<k$, we have by Theorem 4.3 that $\infty$ is an unstable value of $h_{j m}$. Therefore, by Lemma 3.9, there is a sequence $h_{m}: X \rightarrow S^{k} \backslash\{\infty\}(m=1,2, \ldots)$ of continuous functions that converges pointwise to $h$ on $E$. Lemma 6.2 completes the proof.

Finally, the proof of Theorem 1.3 is a straightforward application of Lemma 6.3 and the Tietze Extension Theorem.

\section{References}

[1] Bagemihl, F., and J.E. McMillan: Uniform approach to cluster sets of arbitrary functions in a disk. - Acta Math. Acad. Sci. Hungar. 17, 1966, 411-418.

[2] Bageminl, F., and W. Seidel: Some boundary properties of analytic functions. - Math. Z. 61, 1954, 186-199.

[3] Bageminl, F., and W. Seidel: A problem concerning the cluster sets of analytic functions. - Math. Z. 62, 1955, 99-110.

[4] Berman, R., T. Nishiura, and G. Piranian: Uncountable-order sets for radial-limit functions. - Ann. Acad. Aci. Fenn. Ser. A I Math. 14, 1989, 291-313.

[5] Berman, R., and T. Nishiura: Boundary interpolation for inner functions on $\sigma$ dispersed subsets of the unit circle. - J. London Math. Soc. (2) 38, 1988, 463-484.

[6] Brinn, L.W.: Analytic functions with prescribed cluster sets. - Trans. Amer. Math. Soc. 300, 1987, 681-693.

[7] Collingwood, E.F., and A.J. Lohwater: Theory of cluster sets. - Cambridge University Press, Cambridge, 1966.

[8] CurTis, D.W., and R.M. Schori: $2^{X}$ and $\mathcal{C}(X)$ are homeomorphic to the Hilbert cube. - Bull. Amer. Math. Soc. 80, 1974, 927-931. 
[9] CurTis, D.W., and R.M. Schori: Hyperspaces of Peano continua are Hilbert cubes. Fund. Math. 101, 1978, 19-38.

[10] Goffman, C.: Real function theory. - Rinehart and Co., New York, 1953.

[11] Hansell, R.W.: On Borel mappings and Baire functions. - Trans. Amer. Math. Soc. 194, 1974, 195-211.

[12] Himmelberg, C.J., F.S. Van Vleck, and K. Prikry: The Hausdorff metric and measurable selections. - Topology Appl. 20, 1985, 121-133.

[13] Hurewicz, W., and H. Wallman: Dimension theory. - Princeton University Press, Princeton, New Jersey, 1941.

[14] Kuratowski, K.: Topology I. - Academic Press, New York, 1966.

[15] Kuratowski, K.: Topology II. - Academic Press, New York, 1968.

[16] NADler, S.B., JR.: Hyperspaces of sets. - Marcel Dekker, Inc., New York, 1978.

[17] Rogers, C.A.: Functions of the first Baire class. - J. London Math. Soc. (2) 37, 1988, 535-544.

Robert D. Berman

Wayne State University

Department of Mathematics

Detroit, MI 48202

U.S.A.

Received 19 March 1991
Togo Nishiura

Wayne State University

Department of Mathematics

Detroit, MI 48202

U.S.A. 